\title{
Uso de mezclas de azoxistrobina y triazoles para controlar enfermedades de fin de ciclo de la soja
}

\author{
Marcelo Carmona ${ }^{1}$, Marcela Gally ${ }^{1}$, Francisco Sautua $^{1}$, Andrés Abello $^{1} \&$ Pedro Lopez $^{1}$
}

${ }^{1}$ Cátedra de Fitopatología. FAUBA. Av. San Martín 4453. Capital Federal, Bs As, Arg. Tel. 4524-8063.

Autor para correnpondência: Marcelo Carmona (carmonam@agro.uba.ar)

Data de chegada: 26/12/2008. Aceito para publicação em: 16/02/2011.

\section{RESUMEN}

Carmona, M.; Gally, M.; Sautua, F.; Abello, A.; Lopez, P.. Uso de mezclas de azoxistrobina y triazoles para controlar enfermedades de fin de ciclo de la soja. Summa Phytopathologica, v.37, n.2, p.134-139, 2011.

Las enfermedades de fin de ciclo de la soja (EFC), ampliamente difundidas en Argentina, afectan el rendimiento del cultivo y la calidad de la semilla cosechada. La aplicación foliar de fungicidas representa una alternativa eficiente en las actuales condiciones de cultivo (monocultivo y siembra directa). Los objetivos del presente trabajo fueron 1) determinar los agentes causales de la EFC presentes en los ensayos, 2) evaluar la disminución de rendimiento causada por las EFC y 3) valorar la eficiencia de control de mezclas de triazoles y azoxistrobina aplicados en las etapas reproductivas R3 o R5, para el control de las mismas. Se realizaron cuatro ensayos, dos en la campaña 2004/2005, y dos en la campaña 2005/2006, en
Armstrong, Santa Fe, núcleo del área sojera de la Región Pampeana. Se utilizaron dos mezclas de azoxistrobina, una con cyproconazole y otra con difenoconazole, y una mezcla de los dos últimos. Los patógenos detectados fueron: Cercospora kikuchii, Colletotrichum truncatum, Septoria glycines, Glomerella glycines y Phomopsis sojae. Todos los tratamientos tuvieron mayores rendimientos que el control no tratado en todos los ensayos, con un nivel de significancia del $5 \%$. Se concluye que, bajo las condiciones de los ensayos, con altas precipitaciones entre R1 y R5.5, los productos utilizados, controlaron eficientemente las EFC presentes.

Palabras clave: fungicidas, triazoles, estrobilurinas, enfermedades de la soja

\section{ABSTRACT}

Carmona, M.; Gally, M.; Sautua, F.; Abello, A.; Lopez, P.. Use of azoxystrobin and triazoles mixtures to control late season diseases in soybean crop. Summa Phytopathologica, v.37, n.2, p.134-139, 2011.

Widely spread in Argentina, late season diseases (LSD), cause yield losses and seed quality changes. Leaf application of fungicides is an effective procedure to manage LSD under the current cropping conditions (monocrop and no-till system). The aims of the present study were 1) to determine causal agents of LSD, 2) to evaluate yield reduction caused by LSD and 3) to evaluate the efficiency of mixtures containing triazoles and strobirulins, sprayed during R3 or R5 reproductive stages to control LSD. Four experiments were carried out in a randomized complete block design, with four replicates in Armstrong, Santa Fe, Argentina, in a soybean cultivation area in the
Pampeana Region. Two assays were performed in 2004/2005, and the remaining ones in 2005/2006 growing seasons. Two azoxystrobin mixtures, one with cyproconazole and the other with difenoconazole, and a mixture of the latter two were tested. The following pathogens were detected : Cercospora kikuchii, Colletotrichum truncatum, Septoria glycines, Glomerella glycines y Phomopsis sojae. All treatments presented higher yield than control, with $5 \%$ of statistic significance. It can be concluded that, under the present experimental conditions, characterized by frequent rains between R1 and R5.5, the tested mixtures efficiently controlled LSD.

Keywords: fungicides, triazoles, strobilurins, soybean diseases.

El monocultivo en siembra directa y el uso de cultivares con alta uniformidad genética han afectado la sanidad de los cultivos de soja (Glycine max L. (Merr)) en Argentina, con un notable incremento tanto en el espectro de enfermedades presentes, así como en la intensidad de las mismas. Las enfermedades de fin de ciclo (EFC), de alta prevalencia en el país, representan una significativa limitante de los rendimientos. Presentan sus síntomas más evidentes en las etapas de generación del rendimiento, aceleran la senescencia de las plantas y disminuyen el número y peso de los granos y, algunas de ellas también la calidad de la semilla. (2, 4, 9, 22). Este grupo de enfermedades, cuyos síntomas se manifiestan en estados reproductivos intermedios y avanzados del cultivo, causan sus daños a través de la disminución del área foliar sana, defoliación, madurez anticipada del cultivo e infección de las semillas $(12,20)$.

Las EFC de mayor prevalencia en Argentina son: tizón de la hoja y mancha púrpura de la semillas (Cercospora kikuchii (T. Matsu \& Tomoyasu) Gardner), antracnosis (Glomerella glycines (Hori)Lehman \& Wolf; Colletotrichum truncatum (Schw.) Andrews \& WD Moore), mancha marrón (Septoria glycines Hemmi), tizón de la vaina y tallo (Phomopsis sojae Lehman), mildiu (Peronospora manshurica 
(Naum.) Syd: Gäum), mancha ojo de rana (Cercospora sojina Hara), mancha anillada (Corynespora cassiicola (Berk \& Curt.) Wei), mancha foliar por Alternaria (Alternaria spp.), pústula bacteriana (Xanthomonas campestris pv glycines (Nakano) Dye.) y tizón bacteriano (Pseudomonas siringae pv glycinea (Coerper) Young, Dye $\&$ Wilkie).

Las principales estrategias para el manejo eficiente de las EFC incluyen el uso de cultivares tolerantes y la rotación de cultivos, ya que la mayoría de los agentes etiológicos de las mismas sobreviven en los rastrojos (16). Además, el tratamiento de semillas con fungicidas es una medida eficiente y complementaria, principalmente en aquellos lotes donde aún no se han observado síntomas de las enfermedades, o en zonas nuevas de cultivo. El control químico durante el cultivo a través de la pulverización de fungicidas en los órganos aéreos, es una medida de control rápida y eficiente, cada vez más difundida en Argentina, ya que gran parte de la producción sojera se hace bajo monocultivo con presencia de rastrojo de soja en superficie, y la mayoría de los cultivares son susceptibles a las $\operatorname{EFC}(5,11)$. De esta manera, la importancia de las EFC en soja justifica la estrategia de utilizar fungicidas para evitar daños y pérdidas y por ello resulta necesario determinar la eficiencia de diferentes moléculas y sus mezclas para este complejo fúngico.

Los productos usados con mayor frecuencia se encuentran dentro de los grupos de los bencimidazoles, los triazoles y las estrobilurinas. En los últimos años, se ha difundido la utilización de mezclas de fungicidas debido a la posibilidad de ejercer mejor control frente a la aparición conjunta de EFC y roya asiática de la soja (Phakopsora pachyrhizi H. Sydow \& Sydow) y para disminuir los riesgos de generación de resistencia a través de la acción combinada de diferentes moléculas químicas $(6,17)$.

Los objetivos del presente trabajo fueron 1) determinar los agentes causales de la EFC presentes en los ensayos bajo estudio, 2) evaluar la disminución de rendimiento causada por las EFC y 3) valorar la eficiencia de control de mezclas de triazoles y azoxistrobina (estrobilurina) aplicados en dos momentos diferentes dentro de las etapas reproductivas del cultivo: R3 (estado de inicio de formación de vainas) o R5 (comienzo del llenado del grano (10).

\section{MATERIALES Y MÉTODOS}

Se llevaron a cabo cuatro ensayos en la localidad de Amstrong (Santa Fe), en dos campañas consecutivas (dos ensayos por temporada), en dos campos situados a $20 \mathrm{~km}$ de distancia, en lotes bajo monocultivo de soja. Se utilizó un cultivar del grupo IV implantado bajo sistema de siembra directa. El diseño experimental de los experimentos fue en bloques completos aleatorizados con 4 repeticiones. El tamaño de las parcelas fue de $20 \mathrm{~m}^{2}$ (10 m x $\left.2 \mathrm{~m}\right)$.

Los tratamientos aplicados consistieron en:

\section{Campaña 2004/2005:}

1. Azoxistrobina + cyproconazole, $300 \mathrm{cc} / \mathrm{ha}+$ aceite mineral parafínico, $500 \mathrm{cc} / \mathrm{ha}$ en R3.

2. Azoxistrobina + difenoconazole, $300 \mathrm{cc} / \mathrm{ha}$ en R3.

3. Cyproconazole + difenoconazole, $250+150 \mathrm{cc} / \mathrm{ha}$ en R3.

4. Azoxistrobina + cyproconazole, $300 \mathrm{cc} / \mathrm{ha}+$ aceite mineral parafínico, $500 \mathrm{cc} / \mathrm{ha}$ en R5.

5. Azoxistrobina + difenoconazole, $300 \mathrm{cc} / \mathrm{ha}$ en R5.

6. Cyproconazole + difenoconazole, $250+150 \mathrm{cc} / \mathrm{ha}$ en R5.

7. Testigo sin aplicacion

\section{Campaña 2005/2006:}

8. Azoxistrobina + cyproconazole, $300 \mathrm{cc} / \mathrm{ha}+$ aceite mineral parafínico, $500 \mathrm{cc} / \mathrm{ha}$ en R3

9. Cyproconazole + difenoconazole, $250+150 \mathrm{cc} / \mathrm{ha}$ en R3

10. Azoxistrobina + cyproconazole, $300 \mathrm{cc} / \mathrm{ha}+$ aceite mineral parafínico, $500 \mathrm{cc} / \mathrm{ha}$ en R5

11. Cyproconazole + difenoconazole, $250+150 \mathrm{cc} / \mathrm{ha}$ en R5

12. Testigo sin aplicacion

Las aplicaciones se realizaron con pulverizador de precisión, con presión constante generada por dióxido de carbono, con pastillas tipo cono hueco y un volumen de caldo de 150 1/ha.

Con el fin de determinar los patógenos involucrados en la sintomatología se incubó el material vegetal con y sin síntomas (hojas, tallos y vainas), previamente desinfectado con hipoclorito al $1 \%$ durante 1 minuto, en cámara húmeda a temperatura entre 24 y $27^{\circ} \mathrm{C}$, con alternancia de 12 horas de luz cercana al ultravioleta y 12 horas de oscuridad.

Se estimó visualmente la severidad estimando el porcentaje de área foliar clorótica, necrosada o con síntomas de las plantas de las dos hileras centrales de cada parcela al estado R5.5 Para la determinación de la eficiencia de control se aplicó la siguiente fórmula:

$$
\% \text { de control }=\frac{\text { Severidad en el testigo }(\%)-\text { Severidad del tratamiento }(\%)}{\text { Severidad en el testigo }(\%)} \times 100
$$

A cosecha se determinaron el rendimiento y sus componentes (número de granos por unidad de superficie y peso de 1000 semillas). Para ello se cosecharon manualmente $8,32 \mathrm{~m} 2$ de cada parcela y se procedió a la cuantificación de los componentes

Las precipitaciones fueron registradas a través de pluviómetros convencionales ubicados en los lotes que estaban instalados los ensayos. Los mismos fueron promediados y contrastados contra el promedio de los últimos 12 años de la localidad más cercana (Marcos Juárez) a la localidad donde estaban los ensayos (Amstrong).

Los datos promedios fueron obtenidos del INTA, Marcos Juárez.

Se analizaron estadísticamente los datos, para establecer una posible interacción entre la mezcla de fungicida aplicada y el momento de aplicación a traves de un analisis de varianza (ANOVA) factorial. Posteriormente se analizó cada uno de los factores por separado. Las medias fueron comparadas por test de Tuckey con un nivel de significancia de $5 \%$. Los datos de severidad fueron transformados con la transformación angular (arcsen “\%).

\section{RESULTADOS}

Los patógenos predominantes que fueron asociados con los síntomas evaluados coincidieron en ambos ensayos. Los más frecuentes en tejidos sintomáticos fueron: en hojas : C. kikuchii y G. glycines y S. glycines; en tallos $P$. sojae, $C$. truncatum y $C$. kikuchii, y en vainas, C. kikuchii, $C$. truncatum; G. glycines y $P$. sojae. En tejido foliar asintomático se detectaron recurrentemente a partir de R3: C. truncatum, G. glycines y P. sojae.

En relación al análisis estadístico, la interacción entre momento de aplicación y fungicida no resultó estadísticamente significativa en ninguno de los cuatro ensayos, por lo cual se realizó el análisis de ambas variables en forma independiente. Todos los tratamientos 
tuvieron mayores rendimientos que el control no tratado en todos los ensayos Los resultados de cada uno de los ensayos se detallan a continuación.

\section{Ensayo 1}

Todos los tratamientos con fungicidas superaron en rendimiento ( $\mathrm{p}<0,0001)$, número de granos por metro cuadrado $(\mathrm{p}=0,0029)$ y peso de mil semillas $(<0,0001)$ al control no tratado (tabla 1$)$. Los tratamientos en ambos momentos de aplicación presentaron rendimiento ( $\mathrm{p}=0,0002$ ), número de granos por metro cuadrado $(\mathrm{p}=0,0078)$ y peso de mil semillas $(<0,0001)$ superiores al testigo (cuadro 2), prácticamente sin diferencias entre ellos.

En todas las parcelas se observaron valores de severidad foliar menores que el testigo ( $p<0,0001)$. La evaluación en R5.5, 20 días después de las aplicaciones, mostró un porcentaje de control de $47 \%$ para las aplicaciones en R3 y de $23 \%$ para las correspondientes a R5 (tabla 2). Se destaca la elevada severidad registrada en el testigo al final del ciclo del cultivo, los valores expresados en las tablas incluyen clorosis y necrosis.

\section{Ensayo 2}

Todos los tratamientos tuvieron rendimientos $(\mathrm{p}=0,0004)$ y peso de mil semillas $(\mathrm{p}<0,0001)$ significativamente superiores respecto del control no tratado (tabla 1). La mayoria de las mezclas usadas presentaron mayor número de granos por metro cuadrado respecto el testigo $(\mathrm{p}=0,0034)$. Ambos momentos de aplicación presentaron rendimiento ( $\mathrm{p}=0,0025$ ), número de granos por metro cuadrado $(\mathrm{p}=0,0098)$ y peso de mil semillas $(<0,0001)$ superiores al testigo (tabla 2), sin diferencias entre ellos (excepto el número de granos en R5).

De la misma manera que en el ensayo 1, todas las parcelas tratadas con fungicidas presentaron valores de severidad foliar menores que el testigo ( $\mathrm{p}<0,0001$ ). La evaluación en R5.5, después de las aplicaciones, mostró un porcentaje de control que varió del $68 \%$ para las aplicaciones en R3 y del $45 \%$ para las correspondientes a R5 (tablas 2).

\section{Ensayo 3}

Ambas mezclas de fungidas tuvieron rendimientos $(\mathrm{p}=0,0011)$ y peso de mil granos ( $\mathrm{p}=0,0044)$ superiores al testigo (tabla 3 ). Ambos momentos de aplicación presentaron rendimiento $(\mathrm{p}=0,0019)$ y peso de mil granos $(\mathrm{p}=0,0038)$ superiores al testigo (tabla 4$)$, sin diferencias entre ellos. Todas las parcelas tratadas presentaron valores de severidad foliar menores que el testigo ( $p<0,0001)$. La evaluación en R5.5 después de las aplicaciones mostró un porcentaje de control del 44\% para las aplicaciones en R3 y del $30 \%$ para las correspondientes a R5 (tabla 4).

\section{Ensayo 4}

Ambas mezcals de fungidas tuvieron rendimientos $(\mathrm{p}=0,0005)$ y número de granos por metro cuadrado $(\mathrm{p}=0,0038)$ superiores al testigo (tabla 3 ). Ambos momentos de aplicación presentaron rendimiento $(\mathrm{p}=0,0024)$ superior al testigo (tabla 4$)$, pero sin diferencias

Tabla 1. Evaluación de la eficiencia de la aplicación de las mezcla de fungicidas. Ensayos 1 y 2, (campaña 2004/2005).

\begin{tabular}{|c|c|c|c|c|c|c|c|c|c|c|}
\hline \multirow{3}{*}{$\frac{\text { Fungicidas }}{\text { Azoxistrobina + cyproconazole }}$} & 1 & 2 & 1 & 2 & 1 & 2 & 1 & 2 & 1 & 2 \\
\hline & \multicolumn{2}{|c|}{$\begin{array}{c}\text { Rendimiento } \\
(\mathrm{kg} / \mathrm{ha})^{1}\end{array}$} & \multicolumn{2}{|c|}{$\begin{array}{l}\text { Peso de } 1000 \\
\text { granos }(\mathrm{gr})^{2}\end{array}$} & \multicolumn{2}{|c|}{$\begin{array}{l}\text { Número de } \\
\text { granos } / \mathbf{m}^{23}\end{array}$} & \multicolumn{2}{|c|}{$\begin{array}{c}\text { Severidad } \\
\text { R5,5 }(\%)\end{array}$} & \multicolumn{2}{|c|}{$\begin{array}{c}\text { Control } \\
(\%)^{5}\end{array}$} \\
\hline & $3486 \mathrm{~b}$ & $3561 \mathrm{~b}$ & $136 \mathrm{~b}$ & $146,5 \mathrm{c}$ & $2569 \mathrm{~b}$ & $2435 \mathrm{~b}$ & $60 \mathrm{a}$ & $27 \mathrm{a}$ & 38 & 63 \\
\hline Azoxistrobina + difenoconazole & $3410 \mathrm{~b}$ & $3335 \mathrm{~b}$ & $136,3 \mathrm{~b}$ & $144,8 \mathrm{~b}$ & $2510 \mathrm{~b}$ & $2308 \mathrm{ab}$ & $63 \mathrm{a}$ & $30 \mathrm{a}$ & 34 & 59 \\
\hline Cyproconazole + difenoconazole & $3365 \mathrm{~b}$ & $3395 \mathrm{~b}$ & $136,5 \mathrm{~b}$ & $143,8 \mathrm{~b}$ & $2473 \mathrm{~b}$ & $2362 \mathrm{~b}$ & $65 \mathrm{a}$ & $29 \mathrm{a}$ & 32 & 60 \\
\hline Testigo & $2764 \mathrm{a}$ & $3005 \mathrm{a}$ & $122,3 \mathrm{a}$ & $139,8 \mathrm{a}$ & $2261 \mathrm{a}$ & $2150 \mathrm{a}$ & $96 \mathrm{~b}$ & $72,5 \mathrm{~b}$ & 0 & 0 \\
\hline
\end{tabular}

Letras distintas dentro de las columnas indican diferencias significativas según Test de Tukey (pd"0.05)

1 Se cosecharon y pesaron $8,32 \mathrm{~m}^{2}$ por parcela

2 Se pesó una muestra de 100 granos por parcela

3 Se obtuvo dividiendo rendimiento por peso de mil granos

4 Se estimó visualmente

5 Calculado como [Severidad testigo (\%) - Severidad tratado (\%) ] x $100 /$ Severidad testigo (\%)

Tabla 2. Evaluación del momento de aplicación de los fungicidas. Ensayos 1 y 2, (campaña 2004/2005).

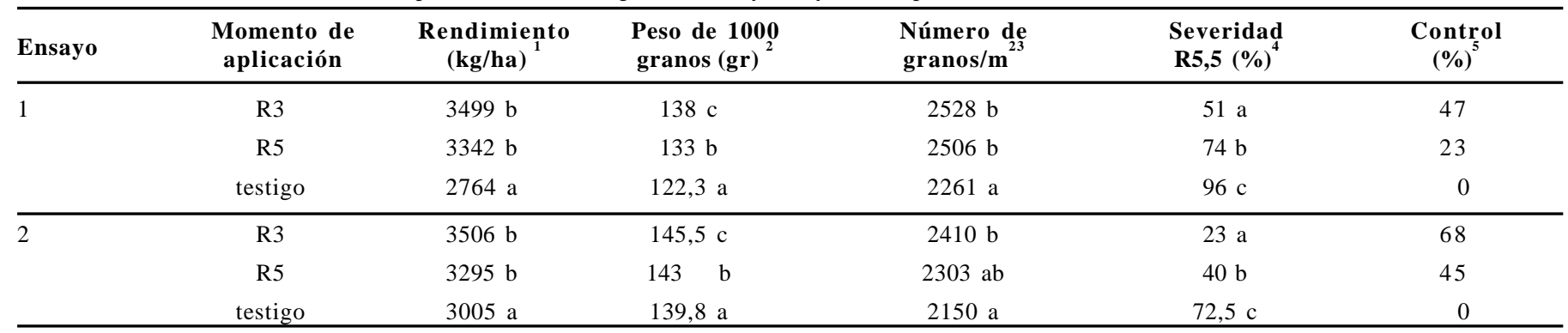

Letras distintas dentro de las columnas indican diferencias significativas según Test de Tukey (pd"0.05)

1 Se cosecharon y pesaron $8,32 \mathrm{~m}^{2}$ por parcela

2 Se pesó una muestra de 100 granos por parcela

3 Se obtuvo dividiendo rendimiento por peso de mil granos

4 Se estimó visualmente

5 Calculado como [Severidad testigo (\%) - Severidad tratado (\%) ] x $100 /$ Severidad testigo (\%) 
significativas para los componentes del mismo. Todas las parcelas tratadas presentaron valores de severidad foliar menores que el testigo ( $\mathrm{p}<0,0001)$. La evaluación en R5.5 después de las aplicaciones mostró un porcentaje de control del $49 \%$ para las aplicaciones en R3 y del 39 $\%$ para las correspondientes a R5 (tabla 4).

\section{DISCUSIÓN}

La mayoría de los patógenos detectados en este estudio son los más comúnmente asociados a las EFC que ocurren en todas las zonas sojeras del país. El desarrollo de las estructuras reproductivas características de los mismos en las incubaciones de tejidos asintomáticos en inicios de R3, indica el prolongado período de latencia de dichos patógenos en los tejidos vegetales, en especial de aquellos que esporulan activamente en condiciones naturales cuando se produce la senescencia de los tejidos, tal es el caso de $C$. truncatum y $G$. glycines, causantes de antracnosis y de $P$. sojae, causante del tizón de vainas y tallos $(2,11,19)$. La presencia de G. glycines (confirmada por Gally y Romero, comunicación personal), teleomorfo de Colletotrichum destructivum, se ha observado tanto en tejidos con síntomas como asintomáticos. Sin embargo las incubaciones de tallos y vainas con síntomas indicaron prevalencia de $C$. truncatum con respecto a $G$. glycines. La prolongada latencia de la mayoría de estos patógenos y la infección conjunta de varios de ellos dificultan la decisión del control químico basada en la observación de síntomas que permitan establecer umbrales (15). Sin embargo, la confirmación de la presencia de los patógenos en las etapas críticas del cultivo en las que se generan los

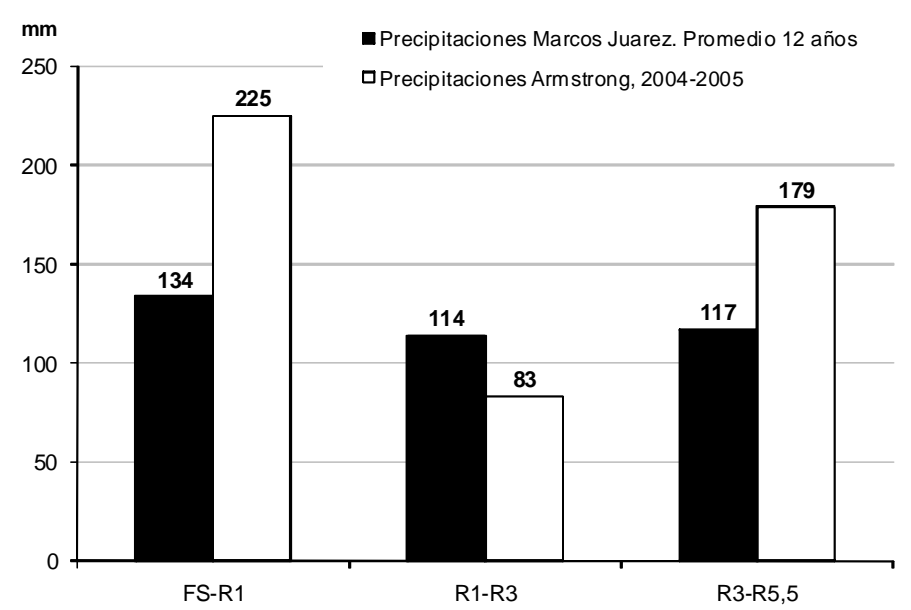

Figura 1. Precipitaciones en Armstrong 2004-2005 vs. promedio 12 años Marcos Juárez. Fecha de Siembra (09/11/04)

componentes del rendimiento (18), determina la necesidad de tomar la decisión del control químico aún cuando los síntomas no se han expresado plenamente. La ocurrencia de lluvias abundantes en los estadios reproductivos que superen los promedios históricos, constituye uno de los factores de mayor influencia en la multiplicación, dispersión e infección por parte de los patógenos, y probablemente se correlacione con la respuesta a la aplicación de los fungicidas (6). En estos 4 ensayos desarrollados, todos los tratamientos químicos generaron un aumento del rendimiento y un

Tabla 3. Evaluación de la eficiencia de la aplicación de las mezcla de fungicidas. Ensayos 3 y 4, (campaña 2005/2006).

\begin{tabular}{|c|c|c|c|c|c|c|c|c|c|c|}
\hline \multirow{3}{*}{ Fungicidas } & 3 & 4 & 3 & 4 & 3 & 4 & 3 & 4 & 3 & 4 \\
\hline & \multicolumn{2}{|c|}{$\begin{array}{c}\text { Rendimiento } \\
(\mathrm{kg} / \mathrm{ha})\end{array}$} & \multicolumn{2}{|c|}{$\begin{array}{c}\text { Peso de } 1000 \\
\text { granos }(\mathrm{gr})^{2}\end{array}$} & \multicolumn{2}{|r|}{$\begin{array}{l}\text { Número de } \\
\text { granos } / \mathrm{m}^{2}\end{array}$} & \multicolumn{2}{|c|}{$\begin{array}{c}\text { Severidad } \\
\text { R5,5 }(\%)^{4}\end{array}$} & \multicolumn{2}{|c|}{$\begin{array}{c}\text { Control } \\
(\%)^{5}\end{array}$} \\
\hline & $4308 \mathrm{~b}$ & $4354 \mathrm{~b}$ & 162 & $164,0 \mathrm{a}$ & 2667 & $2655 \mathrm{~b}$ & $52 \mathrm{a}$ & 39,3 a & 40 & 55 \\
\hline Cyproconazole + difenoconazole & $4229 \mathrm{~b}$ & $4312 b$ & $161,8 \mathrm{~b}$ & $163,3 \mathrm{a}$ & 2616 & $2644 \mathrm{~b}$ & $58 \mathrm{~b}$ & 39,8 a & 33 & 54 \\
\hline testigo & 3925 a & 3876 a & 154 & $161,8 \mathrm{a}$ & 2556 & $2404 \mathrm{a}$ & $87 \mathrm{c}$ & $70,3 \mathrm{~b}$ & 0 & 19 \\
\hline
\end{tabular}

Letras distintas dentro de las columnas indican diferencias significativas según Test de Tukey (pd"0.05)

1 Se cosecharon y pesaron $8,32 \mathrm{~m}^{2}$ por parcela

2 Se pesó una muestra de 100 granos por parcela

3 Se obtuvo dividiendo rendimiento por peso de mil granos

4 Se estimó visualmente

5 Calculado como [Severidad testigo (\%) - Severidad tratado (\%)] x $100 /$ Severidad testigo (\%)

Tabla 4. Evaluación del momento de aplicación de los fungicidas. Ensayos 3 y 4, (campaña 2005/2006).

\begin{tabular}{|c|c|c|c|c|c|c|}
\hline Ensayo & $\begin{array}{l}\text { Momento de } \\
\text { aplicación }\end{array}$ & $\underset{(\mathrm{kg} / \mathrm{ha})}{\operatorname{Rendimiento}}$ & $\begin{array}{l}\text { Peso de } 1000 \\
\text { granos }(\mathrm{gr})\end{array}$ & $\begin{array}{l}\text { Número de } \\
\text { granos/m }\end{array}$ & $\begin{array}{c}\text { Severidad } \\
\text { R5,5 }(\%)^{4}\end{array}$ & $\begin{array}{l}\text { Control } \\
(\%)^{5}\end{array}$ \\
\hline \multirow[t]{3}{*}{3} & $\mathrm{R} 3$ & $4350 \mathrm{~b}$ & $163,3 \mathrm{~b}$ & $2671 \mathrm{a}$ & $49 \mathrm{a}$ & 44 \\
\hline & R5 & $4188 \mathrm{~b}$ & $161 \quad b$ & $2612 \mathrm{a}$ & $61 \mathrm{~b}$ & 30 \\
\hline & testigo & $3925 \mathrm{a}$ & $154 \quad \mathrm{a}$ & $2556 \mathrm{a}$ & $87 \mathrm{c}$ & 0 \\
\hline \multirow[t]{3}{*}{4} & R3 & $4357 \mathrm{~b}$ & $163,5 \mathrm{a}$ & $2667 \mathrm{a}$ & $36 \mathrm{a}$ & 49 \\
\hline & R5 & $4309 \mathrm{~b}$ & $163,8 \mathrm{a}$ & $2632 \mathrm{a}$ & $43 \mathrm{~b}$ & 39 \\
\hline & testigo & $3876 \mathrm{a}$ & $161,8 \mathrm{a}$ & $2404 \mathrm{a}$ & $70 \mathrm{c}$ & 0 \\
\hline
\end{tabular}

Letras distintas dentro de las columnas indican diferencias significativas según Test de Tukey (pd"0.05)

1 Se cosecharon y pesaron $8,32 \mathrm{~m}^{2}$ por parcela

2 Se pesó una muestra de 100 granos por parcela

3 Se obtuvo dividiendo rendimiento por peso de mil granos

4 Se estimó visualmente

5 Calculado como [Severidad testigo (\%) - Severidad tratado (\%)] x 100 / Severidad testigo (\%) 
control eficaz de las EFC, siendo ambos momentos sugeridos para la aplicación (R3 y R5), adecuados para el control de las EFC. Sin embargo, las aplicaciones en $\mathrm{R} 3$, presentaron una tendencia de respuesta superior comparado a R5.

Varios estudios $(4,6)$ estimaron daños promedio en soja en Argentina por efecto de las EFC que oscilaron entre $8 \%$ y $10 \%$, con un máximo individual de $30 \%$. Para las condiciones de estos ensayos, las diferencias de rendimientos entre las parcelas sin tratar y las pulverizadas podrían ser analizadas como los daños que causaron las EFC. Con este razonamiento, se han podido registrar daños o respuesta en el rendimiento de hasta $722 \mathrm{~kg} / \mathrm{ha}$ (Tabla 1, ensayo 1), con un mínimo de hasta $304 \mathrm{~kg} / \mathrm{ha}$ (Tabla 3, ensayo 3).

La magnitud de esta elevada respuesta a la aplicación de fungicidas podría atribuirse principalmente a las precipitaciones ocurridas: las lluvias entre siembra y R1, y entre R3 y R6, superaron al promedio histórico (12 años) de la localidad vecina de Marcos Juárez en la campaña 2004/2005 (Figura 1). Estos registros podrían ser de utilidad para establecer una ayuda o indicador del momento oportuno de aplicación de fungicidas para el control de las EFC

En relación a las moléculas y sus mezclas utilizadas en este trabajo, la aplicación de las mismas proporcionó alta eficacia de control, generando elevados porcentajes de control y aumentos en el rendimiento y sus componentes, cuya magnitud dependió del ensayo y campaña analizada. Semejantes resultados con todas o algunos de estos tratamientos y moléculas fueron obtenidos en el control de las EFC, por da Costa (2005), Alessio (2008), Godoy y Canteri ( 2008) y Camargo y Yuyama (2001). Asimismo varios trabajos recomiendan estas moléculas para el control de la roya asiática $(8,14,21)$ y por lo tanto presentan un comportamiento fungitóxico adicional a $\mathrm{P}$. pachyrhizi que les permite controlar ambas problemáticas fúngicas.

Se concluye que la aplicación de azoxistrobina en mezclas con triazoles, así como la mezcla de los triazoles evaluados en los ensayos, controló las EFC presentes y aumentó el rendimiento, el peso de 1000 semillas, y en algunos casos el número de granos por metro cuadrado. Las aplicaciones de los fungicidas en R3 resultaron ser más eficientes que las efectuadas en R5 para controlar las enfermedades presentes. No se observaron diferencias entre los fungicidas utilizados en estos experimentos. Las precipitaciones fueron superiores al promedio histórico para la zona, lo cual indicaría que las mayores lluvias estimularían los hongos causantes de estas enfermedades ocasionando daños y pérdidas.

Más investigaciones que involucren estudios de variables ambientales y diferentes moleculas y mezclas, serán necesarias para desarrollar una recomendación que pueda ser práctica y útil para el manejo químico de estas enfermedades.

\section{AGRADECIMIENTOS}

Investigación subsidiada por UBACyT G020, G043 y Syngenta.

\section{REFERENCIAS BIBLIOGRAFICAS}

1. Alessio, D. Momentos e número de aplicações de fungicidas e seu efeito sobre a duração da área foliar sadia e o rendimento de grãos em soja. 2008. Tese de Mesttre Universidade de Passo Fundo, RS.

2. Arias, N.; Pelossi, N.; De Battista, J.; Carmona, M. Control químico de enfermedades de fin de ciclo en soja. Cultivo de soja en el centro este de entre Ríos, Resultados 2002-2003. Boletin Técnico INTA EEA Concepción del Uruguay, n. 44, p. 57-63, 2003.

3. Camargo, T. V.; Yuyama, M. M. Controle das doenças de final de ciclo da soja (Septoria glycines e Cercospora Kikuchii) com benomyl. Fitopatologia Brasileira, Lavras, v. 26, p.330, 2001.

4. Carmona, M.; Gally, M.; Grijalba, P.; Sugia, V.; Jaeggi, E. Frequency and chemical control of causal pathogens of soybean late season diseases in the Pampeana egion. In: World Soybean Research Conference, $7^{\circ}$; International Soybean Processing and Utilization Conference, $4^{\circ}$, Congresso Mundial de Soja, 3ㅜ, 2004, Foz de Iguazú. Abstracts: Foz de Iguazú, EMBRAPA, 2004. p. 159.

5. Carmona, M. Enfermedades de fin de ciclo y roya asiática de la soja. Un análisis de sus daños y el uso estratégico de fungicidas. In: Jornada Regional de Fungicidas y Tecnología de Aplicación del Cono Sur, $1^{\circ}, 2005$, Rosario, Argentina. Actas, Buenos Aires.: Technidea , 2005. p. 49-64.

6. Carmona, M. Importancia de las enfermedades de fin de ciclo: su relación con la ecofisiología y el uso estratégico de fungicidas en el cultivo de soja. In: Workshop de enfermedades de hoja, tallo y raíz en Mercosoja 2006, 3er Congreso de Soja del Mercosur, 2006, Rosario. Actas. Rosario: ACSOJA, 2006. p. 321-324.

7. da Costa, D.I.F. Controle de doenças de final de ciclo na cultura da soja. 2005. Tese de Doutorado. Universidade Federal de Santa Maria. Santa Maria RS.

8. da Silva, J. F.; Juliatti, F. C.; Rezende, A. A. Diferentes épocas de aplicação de azoxistrobina + ciproconazole seguida de ciproconazole no controle da ferrugem asiática. Bioscience Journal, Uberlândia, v. 26, n. 2, p. 216-225, 2010.

9. Díaz, C. G.; Ploper, L. D.; Galvez, M. R.; Gonzalez, V.; Zamorano, M .A.; Jaldo, H. E.; Lopez, C.; Ramallo, J. C. Efecto de las enfermedades de fin de ciclo en el crecimiento de distintos genotipos de soja relacionado a la fecha de siembra. Agriscientia, Córdoba, v. 22, n. 1, p. 1-7, 2005.

10. Fehr, W.R.; Caviness, C.E. Stages of soybean development. Special Report, Iowa, n. 80, p. 11, 1977.

11. Gally, M. Enfermedades de fin de ciclo de la soja en la Región Pampeana. In: Jornadas Técnicas de Manejo integrado de enfermedades en cultivos extensivos, 2003, Bs. As. Actas: Buenos.Aires: Technidea, 2003. p. 77-80.

12. Gally, M.; Carmona, M.; Grijalba, P.; Barreto, D.; Sugía, V. Control of soybean seed-borne pathogens by foliar fungicide applications in Argentina. In: ISTA Congress Seed Symposium, 27, 2004, Budapest. Proceedings. Budapest: ISTA, 2004. p. 110.

13. Godoy, C.V. ; Canteri, M.G. Efeito da severidade de oídio e crestamento foliar de cercospora na produtividade da cultura da soja. Fitopatologia Brasileira, Lavras, v. 29, p.526-531, 2004.

14. Godoy, C.V., Canteri, M.G. Efeitos protetor, curativo e erradicante de fungicidas no controle da ferrugem da soja causada por Phakopsora pachyrhizi, em casa de vegetação. Fitopatologia Brasileira, Lavras, v. 29, p.097-101, 2004.

15. Guerzoni, R.A. Efeito das doencas foliares de final de ciclo (Septoria glycines e Cercospora kikuchii) na duracao da area foliar sadia da soja. 2001. 49f. Tesis (Mestrado em Fitopatologia). Escola Superior de Agricultura Luiz de Queiroz, Universidade de Sao Paulo, Piracicaba.

16. Hoffmann, L.; Reis, E.; Forcelini, C.; Panisson, E.; Mendes, C.; Casa, R. Efeitos da rotação de cultura, de cultivares e da aplicação de fungicida sobre o rendimento de grãos e doenças foliares em soja. Fitopatologia Brasileira, Brasília, v. 29, p. 245-251, 2004.

17. Ivancovich, A. Soybean rust in Argentina. Plant Disease, St. Paul, v. 89, n. 6, p. 667-668, 2005.

18. Kantolic, A.; Carmona, M. Bases ecofisiológicas de la generación de rendimiento: relación con el efecto de las enfermedades foliares y el uso de fungicidas en el cultivo de soja. 1.ed. Buenos Aires: FAUBA, 2005. 24p.

19. Klingelfuss, L.; Yorinori, J.T. Infecçao latente de Colletotrichum truncatum e Cercospora kikuchii em soja. Fitopatología Brasileira, Brasilia, v. 26, p. 158-164, 2001.

20. Molina, J.; Carmona, M.; Babbitt, S.; Gally, M.; Sugía, V.; Grijalba, P. Effect of foliar fungicides application on soybean seed quality. In: ISTA Congress Seed Symposium, 27, 2004, Budapest. Proceedings. Budapest: ISTA, 2004. p. 53. 
21. Navarini, L.; Dallagnol, L. J.; Balardin, R. S.; Moreira, M. T.; Meneghetti, R. C.; Madolosso, M. G. Controle químico da ferrugem asiática (Phakopsora pachyrhizi Sydow) na cultura da soja. Summa Phytopathologica, Jaboticabal, v. 33, n. 2, p.182-186, 2007.
22. Wrather, J.A.; Anderson, T.R.; Arsyad, D.M.; Tang, Y.; Ploper, L.D.; Porta- Puglia, A.; Ram, H.H.; Yorinori, J.T. Soybean disease loss estimates for the top ten soybean-producing countries in 1998. Canadian Journal of Plant Pathology, Ottawa, v. 23, p. $115-121,2001$. 